OPEN ACCESS

Edited by:

Georgios Tsivgoulis,

National and Kapodistrian University of

Athens, Greece

Reviewed by:

Lina Palaiodimou,

University General Hospital Attikon,

Greece

Aikaterini Theodorou,

University General Hospital Attikon,

Greece

${ }^{*}$ Correspondence:

Jan Viteček

jan.vitecek@ibp.cz

Specialty section:

This article was submitted to

Cardiovascular and Smooth Muscle

Pharmacology,

a section of the journal

Frontiers in Pharmacology

Received: 14 July 2021

Accepted: 27 August 2021

Published: 15 September 2021

Citation:

Thalerová S, Pešková M, Kittová P,

Gulati S, Viteček J, Kubala $L$ and Mikulik R (2021) Effect of Apixaban Pretreatment on Alteplase-Induced

Thrombolysis: An In Vitro Study.

Front. Pharmacol. 12:740930.

doi: 10.3389/fphar.2021.740930

\section{Effect of Apixaban Pretreatment on Alteplase-Induced Thrombolysis: An In Vitro Study}

\author{
Sandra Thalerová ${ }^{1,2,3}$, Michaela Pešková ${ }^{2}$, Patrícia Kittová $^{2}$, Sumeet Gulati ${ }^{1,2}$, \\ Jan Viteček ${ }^{2,4 *}$, Lukáš Kubala ${ }^{2,4}$ and Robert Mikulik ${ }^{1}$
}

${ }^{1}$ Neurology Department, International Clinical Research Center, St. Anne's University Hospital Brno, Brno, Czechia, ${ }^{2}$ Institute of Biophysics of the Czech Academy of Sciences, Brno, Czechia, ${ }^{3}$ Department of Biochemistry, Faculty of Science, Masaryk University, Brno, Czechia, ${ }^{4}$ Center of Biomolecular and Cell Engineering, International Clinical Research Center, St. Anne's University Hospital Brno, Brno, Czechia

Benefit of thrombolytic therapy in patients with acute stroke, who are on anticoagulant treatment, is not well addressed. The aim of this study was to investigate whether apixaban can modify the thrombolytic efficacy of alteplase in vitro. Static and flow models and two variants of red blood cell (RBC) dominant clots, with and without apixaban, were used. Clots were prepared from the blood of healthy human donors and subsequently exposed to alteplase treatment. Apixaban and alteplase were used in clinically relevant concentrations. Clot lysis in the static model was determined both by clot weight and spectrophotometric determination of RBC release. Clot lysis in the flow model was determined by measuring recanalization time, clot length and spectrophotometric determination of RBC release. In the static model, clots without apixaban; compared to those with apixaban had alteplaseinduced mass loss $54 \pm 8 \%$ vs. $53 \pm 8 \%, p=1.00$; RBC release $0.14 \pm 0.04$ vs. $0.12 \pm 0.04$, $p=0.14$, respectively. Very similar results were obtained if plasma was used instead of physiological buffered saline as the incubation medium. In the flow model, clot lysis without apixaban; compared to those with apixaban was as follows: recanalization time $107 \pm$ 46 min vs. $127 \pm 31$ min, $p=1.00$; recanalization frequency $90 \pm 22 \%$ vs. $90 \pm 22 \%$, $p=1.00$; clot volume reduction $32 \pm 15 \%$ vs. $34 \pm 10 \%, p=1.00$; RBC release $0.029 \pm$ 0.007 vs. $0.022 \pm 0.007, p=0.16$, respectively. Apixaban had no positive effect on alteplaseinduced thrombolysis in both the in vitro static and flow models. Our data support current clinical practice, such that thrombolysis is contraindicated in stroke treatment for patients who have been treated with anticoagulants.

Keywords: alteplase, apixaban, clot, in vitro, thrombolysis, stroke

\section{INTRODUCTION}

Non-vitamin K antagonist oral anticoagulants [novel oral anticoagulants (NOACs)] are the preferable option over warfarin for secondary prevention of thrombotic events in patients with atrial fibrillation (Connolly et al., 2009; Granger et al., 2011; Patel et al., 2011). Their use in clinical practices is thus increasing (Jin et al., 2018; Chao et al., 2019). More frequent use, however, of NOACs

Abbreviations: FXa, factor Xa; NOACs, novel oral anticoagulants; PBS, physiological buffered saline; RBC, red blood cell. 
itself poses new challenges in patients subsequently suffering from stroke. While on NOACs, thrombolytic treatment is usually contraindicated (Tsivgoulis and Safouris, 2017; Šaňák et al., 2018; Shahjouei et al., 2020; Tsivgoulis et al., 2021). The only alternative, mechanical thrombectomy, is however, suitable only for patients with large vessel occlusion and is not universally available (Šaňák et al., 2018; Shahjouei et al., 2020). Idarucizumab is an antidote that allows thrombolysis in patients on dabigatran (Šaňák et al., 2018). It is not, however, clear if the antidote for direct factor $\mathrm{Xa}$ (FXa) inhibitors, adexanate, will allow thrombolysis in patients on apixaban and/or if the cost of adexanate will prevent its use in many countries around the world. Therefore, it is important to study interaction between intravenous alteplase and NOACs, especially direct FXa inhibitors.

Apixaban is a NOAC, which directly inhibits both free and clot-bound coagulation factor FXa, preventing prothrombin cleavage to active thrombin (Jiang et al., 2009; Connolly et al., 2011; Byon et al., 2019). In vitro studies (Jiang et al., 2009; Escolar et al., 2013) have demonstrated that, by interfering with thrombin generation, apixaban prolongs clotting time and clot formation time, thereby altering viscoelastic parameters during clot formation. Animal models have also shown that apixaban reduces platelet and fibrin formations on damaged vessels (Escolar et al., 2013) and inhibits clot formation in a dose-dependent manner (Pinto et al., 2007). Recent studies (Farag et al., 2016; Carter et al., 2018; Spinthakis et al., 2019) have also indicated that apixaban shortens fibrinolysis times and enhances endogenous fibrinolysis in patients.

Despite the efficiency of apixaban pretreatment in high-risk stroke patients, its interaction with thrombolytics is not well known (Ishihara et al., 2014; Seiffge et al., 2015; Jin et al., 2018; Chao et al., 2019). In order to provide a better understanding, we established a combination of two in vitro thrombolytic models to investigate the interaction between apixaban pretreatment and alteplase-induced thrombolysis.

\section{METHODOLOGY}

This is an in vitro study using both static and flow models to study the effect of apixaban pretreatment on alteplase-induced thrombolysis and recanalization using apixaban-pretreated human blood clots.

\section{Treatment Groups}

Four experimental groups were established to test the hypothesis that apixaban-pretreatment with subsequent alteplase-treatment had different outcome, as compared to alteplase-treatment of clots. These four groups included: "untreated" (subject not on apixaban, without thrombolytic treatment), "alteplase-treated" (subject not on apixaban, with thrombolytic treatment), "apixaban-pretreated" (subject on apixaban, without thrombolytic treatment) and "apixabanpretreated + alteplase-treated" (subject on apixaban, with thrombolytic treatment).

\section{In Vitro Model}

Experiments were performed in static and flow in vitro models described in contemporary literature (Harpaz et al., 1993; Prasad et al., 2006), which were optimized to determine the suitably measurable effect of alteplase in highly repeatable manner. Briefly, the static model consisted of $1.5 \mathrm{ml}$ plastic tubes (Eppendorf, Germany) filled with medium to a total volume of $500 \mu \mathrm{L}$, in which the clots were individually incubated. Tubes were placed into a dry-block incubator at $37^{\circ} \mathrm{C}$ and incubation lasted $60 \mathrm{~min}$ (the same amount of time indicated for alteplase treatment of stroke patients). The flow model comprised of silicone chips (Sylgard 184 Silicone Elastomer, Dow Corning, United States) prepared according to human middle cerebral artery anatomy, with narrowings dimensionally based on patient CT scans $(n=4)$. The bifurcation was included to enable permanent circulation in the system. Each silicone chip was connected by plastic pipes (internal diameter $3.1 \mathrm{~mm}$ ) to an 8channel pump head peristaltic pump (Gilson Minipuls 3, Gilson, Inc., United States) and the whole system was maintained at $37^{\circ} \mathrm{C}$ for incubation lasting $180 \mathrm{~min}$. This arrangement maintained the hydrodynamic forces involved in clot removal (Diamond, 1999), whereby a pressure gradient of $10 \mathrm{~mm} \mathrm{Hg}$ was generated across the occlusion.

\section{Clots and Media Preparation}

$\mathrm{RBC}$ dominant clots were employed in both types of in vitro model. In the static model, clots were prepared from $200 \mu \mathrm{L}$ whole human venous blood and clotted in borosilicate glass tubes (internal diameter $8 \mathrm{~mm}$ ) for $5 \mathrm{~h}$ at room temperature to allow for proper retraction (Sutton et al., 2013). Clots were either incubated in physiological buffered saline (PBS, pH 7.4) or 5-fold diluted human plasma. Concerning the flow model, clots were prepared from $100 \mu \mathrm{L}$ blood and clotted in borosilicate glass tubes (internal diameter $6 \mathrm{~mm}$ ) for $4 \mathrm{~h}$ at room temperature. Clots in this instance were incubated in 5fold diluted human plasma only.

Two clots variants were used-those without or; those supplemented with apixaban $\left(250 \mathrm{ng} \mathrm{ml}^{-1}\right.$ ), (a representative of the apixaban-pretreated group). Both variants were divided into two groups: untreated and alteplase-treated $\left(1.3 \mathrm{mg} \mathrm{L}^{-1}\right)$.

All blood donors had agreed to donate blood samples on the premise of signed informed consent for the collection of blood. Individuals who had received acetylsalicylic acid, non-steroidal anti-inflammatory or antiplatelet drugs within 7 days before blood collection were excluded.

All media were prepared using reagent grade chemicals. PBS ( $\mathrm{pH}$ 7.4) contained $8 \mathrm{~g} \mathrm{NaCl}, 2.3 \mathrm{~g} \mathrm{Na}_{2} \mathrm{HPO}_{4} .12 \mathrm{H}_{2} \mathrm{O}, 0.2 \mathrm{~g} \mathrm{KCl}$ and $0.2 \mathrm{~g} \mathrm{KH}_{2} \mathrm{PO}_{4}$ per liter. Plasma was freshly prepared for each experiment from donors' citrated blood (in standard ratio $3.8 \%$ sodium citrate:blood, 1:9) by centrifugation (2000 g, $\left.10 \mathrm{~min}, 4^{\circ} \mathrm{C}\right)$; diluted 5-fold with PBS and kept at $4^{\circ} \mathrm{C}$ prior to the experiment.

\section{Apixaban and Alteplase}

Apixaban (provided by Pfizer Inc., United States; material no. 1151519, batch no. ABA8622) was initially dissolved in dimethyl sulfoxide, and subsequently diluted with PBS to a concentration of $2.5 \mathrm{mg} \mathrm{ml}^{-1}$. Diluted apixaban was stored 
aliquoted at $-20^{\circ} \mathrm{C}$ and was not re-frozen once thawed. Concentration of apixaban was selected to be in line with clinically relevant dosing for the secondary prevention of thrombotic events in patients with atrial fibrillation, which is $250 \mathrm{ng} \mathrm{ml}^{-1}$ (Artang et al., 2017).

Alteplase (Actilyse, provided by Boehringer-Ingelheim International GmbH, Germany; Z. Nr. 1-24,717) was dissolved in distilled water to a concentration of $1 \mathrm{mg} \mathrm{ml}^{-1}$ and was stored aliquoted at $-20^{\circ} \mathrm{C}$ (not re-frozen once thawed); after which it was further diluted with PBS. The final concentration of alteplase was also selected to be in line with clinically relevant dosing indicated for patients with ischemic stroke $\left(1.3 \mathrm{mg} \mathrm{L}^{-1}\right)$, according to the manufacturer's instructions and supporting pharmacokinetic data (Acheampong and Ford, 2012).

\section{Measure of Lytic Efficacy}

Clot lysis in the static model was determined by measuring clot weight (Prasad et al., 2006; Elnager et al., 2014) and by spectrophotometric determination of red blood cell (RBC) release into the incubation media at $575 \mathrm{~nm}$. Clot lysis in the flow model was determined by measuring recanalization time in addition to clot length and spectrophotometric determination of $\mathrm{RBC}$ release. Recanalization frequency was determined as percentage ratio of complete recanalizations to the total number of samples in the given treatment group.

\section{Data Analysis and Statistics}

We expected relative clot mass loss to be $30 \pm 10 \%$ in the untreated group. In the static model, experiments in PBS were performed with two repetitions (with at least a 1-month interval) on samples from 10 healthy male blood donors (aged $31 \pm$ 10 years, ranging from 22 to 53 years) for each variant of the clots (same donors for both clot variants), which would give us $80 \%$ power to detect relative clot mass loss of $43 \pm 10 \%$ or more in the treated group. Experiments in 5-fold diluted plasma were performed on samples from 5 healthy male blood donors (mean age $32 \pm 13$ years, ranging from 23 to 53 years) for each variant of the clots (same donors for both clot variants). All samples were processed in triplicates.

We expected recanalization time to be $180 \pm 25 \mathrm{~min}$ in the untreated group. Flow model experiments were performed on samples from 5 healthy male blood donors (mean age $32 \pm$ 9 years, ranging from 23 to 53 years) for each variant of clots (different donors for clot variants), which would give us $80 \%$ power to detect recanalization time $135 \pm 25 \mathrm{~min}$ or less in the treated group. All samples were processed in duplicates.

All analyses were performed with STATISTICA 12 (StatSoft) software. Data are expressed as mean \pm SD and medians with minimum and maximum values. The forest plot shows mean values and confidence intervals (95\%). Unpaired t-test was used to compare data. Bonferroni correction of $p$ value for multiple-comparisons was applied. The number of null hypotheses considered for static and flow model was 2 and 4, respectively. All $p$-values are reported after Bonferroni correction. $p$-values $\leq 0.05$ were considered to be statistically significant.

\section{RESULTS}

\section{Thrombolysis}

\section{Static Model}

Alteplase treatment provided greater clot mass loss compared to the untreated group ( $54 \pm 8 \%$ vs. $36 \pm 11 \%, p<0.01)$ and greater $\mathrm{RBC}$ release compared to the untreated group $(0.14 \pm 0.04$ vs. $0.07 \pm 0.03, p<0.01)$. For clots without apixaban and with apixaban, alteplase induced the same clot mass loss (54 $\pm 8 \%$ vs. $53 \pm 8 \%, p=1.00)$ and the same RBC release $(0.14 \pm 0.04$ vs. $0.12 \pm 0.04, p=0.14$ ). Additionally, clots without apixaban and with apixaban, both without alteplase treatment showed the same clot mass loss ( $36 \pm 11 \%$ vs. $35 \pm 10 \%, p=1.00)$ and the same RBC release $(0.07 \pm 0.03$ vs. $0.07 \pm 0.03, p=1.00)$. Results were nearly the same if plasma was used instead of PBS. Results are shown in Figures 1, 2 and in Supplementary Tables S1, S2.

\section{Flow Model}

Alteplase treatment provided lower recanalization time compared to the untreated group $(107 \pm 46 \mathrm{~min}$ vs. $180 \pm$ $0 \mathrm{~min}, p<0.01)$ and greater recanalization frequency $(90 \pm$ $22 \%$ vs. $0 \pm 0 \%, p<0.01)$, RBC release $(0.029 \pm 0.007$ vs. $0.012 \pm 0.007, p<0.01)$ and clot volume reduction $(32 \pm 15 \%$ vs. $14 \pm 15 \%, p=0.02$ ). For clots without and with apixaban, alteplase induced the same recanalization time (107 $\pm 46 \mathrm{~min}$ vs. $127 \pm 31 \mathrm{~min}, p=1.00)$, recanalization frequency ( $90 \pm 22 \%$ vs. $90 \pm 22 \%, p=1.00$ ), clot volume reduction ( $32 \pm 15 \%$ vs. $34 \pm$ $10 \%, p=1.00)$ and $\mathrm{RBC}$ release $(0.029 \pm 0.007$ vs. $0.022 \pm 0.007$, $p=0.16)$. Results are shown in Figures 3-6 and in Supplementary Table S3.

\section{Clotting}

Clots prepared from blood without apixaban showed significantly higher weight after 5-h of clotting compared to clots supplemented with apixaban $(0.08 \pm 0.01 \mathrm{~g}$ vs. $0.07 \pm 0.01 \mathrm{~g}$, $p<0.01$ ), (Figure 7). We have not observed any significant impact of such change of clot mass and respective volume in controls as well as alteplase-treated variants.

\section{DISCUSSION}

Our in vitro study aimed to determine whether apixaban pretreatment modifies efficacy of thrombolytic treatment with alteplase. Two in vitro models were used. Both were optimized to detect the lytic effect of alteplase in a highly repeatable manner. The effect of alteplase was observed even with PBS as the incubation medium and efficacy of alteplase was thus intermediated by plasminogen inside the clot. Concentrations of alteplase (and apixaban) were calculated to reflect therapeutic doses used in humans (Acheampong and Ford, 2012; Artang et al., 2017). The extent of the effect of alteplase was comparable to the rate of clot lysis in humans. Thus clot volume reduction median was $33 \%$ at 180 min with alteplase in the flow model, which is similar to the median clot volume reduction of $32 \%$ (Kim et al., 2015) in humans. All these findings support that although 


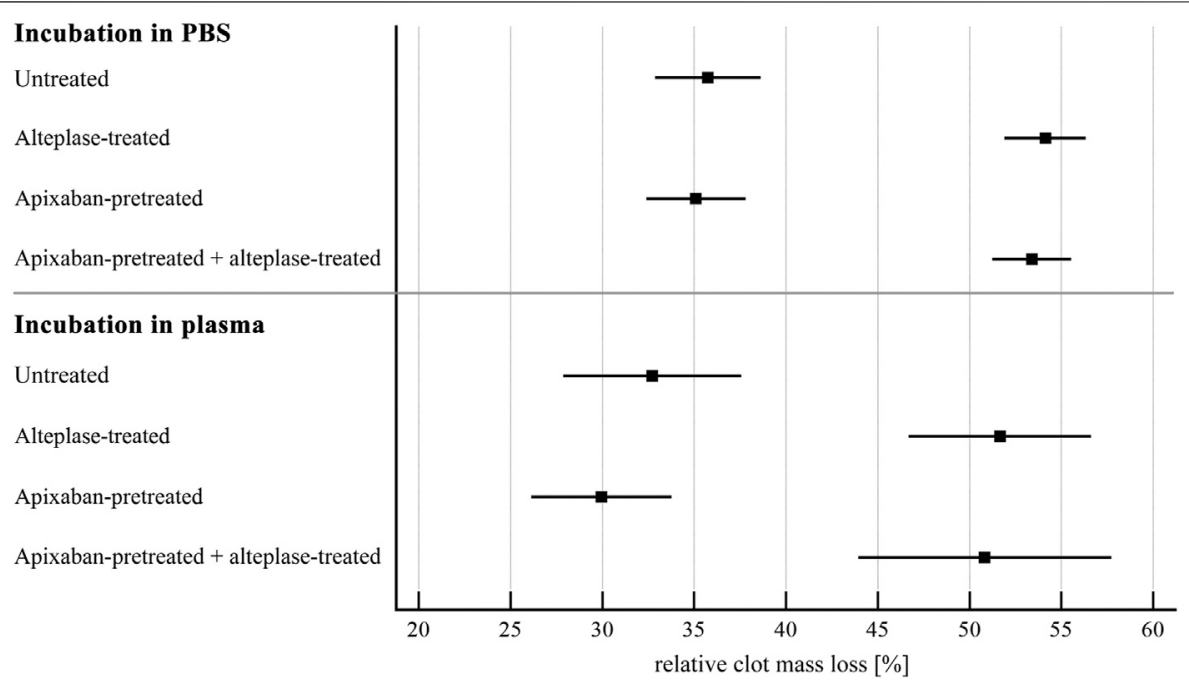

FIGURE 1 | Static model: clot lysis expressed as relative clot mass loss. The forest plot shows mean values (square) and 95\% confidence interval (whiskers). $n=54-57$ for incubation in PBS and 12-15 for incubation in plasma. Results demonstrate that alteplase treatment provided efficient thrombolysis, e.g., documented as greater clot mass loss compared to untreated group in plasma (52 $\pm 9 \%$ vs. $33 \pm 9 \%, p<0.01)$. For clots with and without apixaban, alteplase-induced lysis did not differ, e.g., in plasma clot mass loss was $51 \pm 11 \%$ vs. $52 \pm 9 \%, p=1.00$. See Supplementary Table S1 for more details.

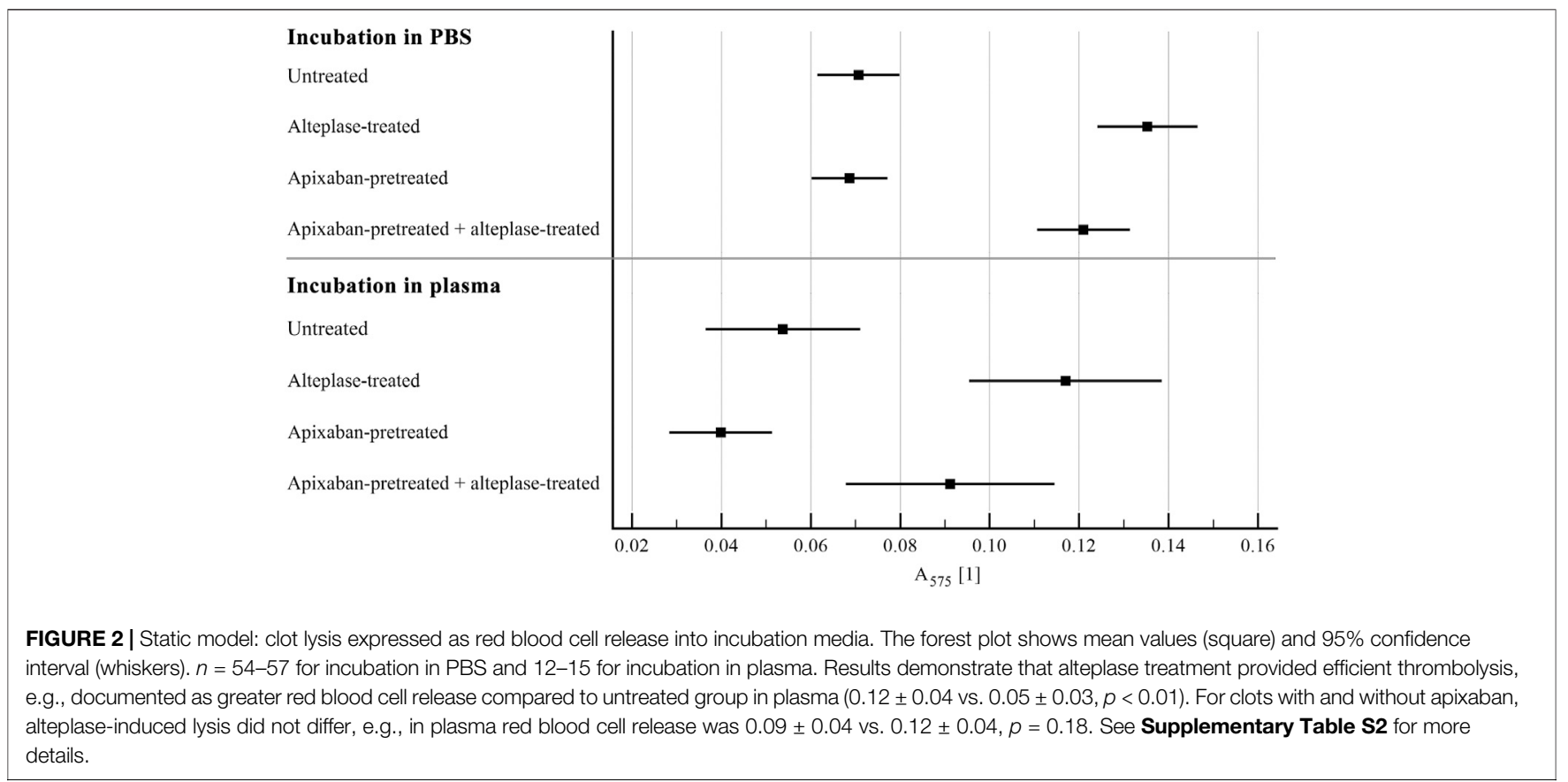

our experiments were conducted in vitro, results may be relevant to clinical data.

The major finding is that apixaban at clinically relevant concentration did not change the level of efficiency of alteplase in vitro. In the static model no significant impact of apixaban on alteplase efficiency was observed. Alteplase lysed apixabanpretreated clots behaved similarly to clots without apixaban pretreatment, both in terms of clot mass loss and RBC release. Spontaneous thrombolysis of these clots remained similar as well.
Minor differences in the initial clot mass did not affect thrombolysis. We reproduced this experiment in the static model in the presence of diluted plasma, since apixaban is a highly hydrophobic compound and plasma proteins could interact with it (Carter et al., 2018). Results were unchanged as compared with PBS. This indicated that all factors important for the alteplase action were already present in the clot and the interaction of apixaban with alteplase was independent of plasma proteins in our experimental system. Compared to previous 
Alteplase-treated

Apixaban-pretreated

Apixaban-pretreated + alteplase-treated

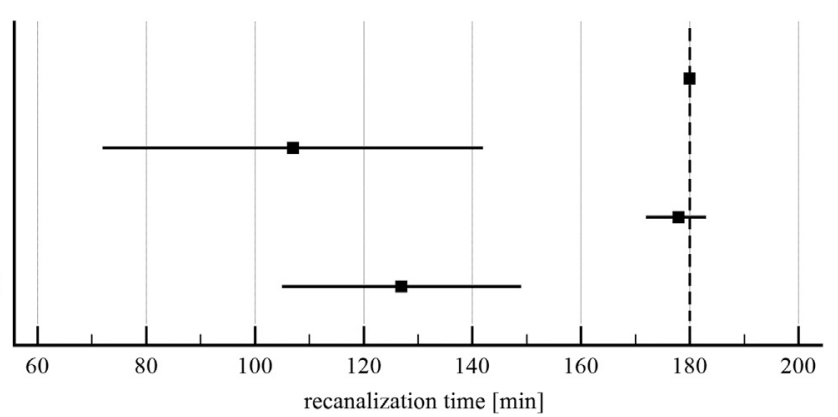

FIGURE 3 | Flow model: time to recanalization of in vitro vessel. The forest plot shows mean values (square) and 95\% confidence interval (whiskers). $n=9-10$. Dashed line shows experiment time window. Results demonstrate that alteplase treatment provided efficient thrombolysis, e.g. documented as lower recanalization time compared to untreated group (107 \pm 46 min vs. $180 \pm 0$ min, $p<0.01$ ). For clots with and without apixaban, alteplase-induced lysis did not differ, e.g. recanalization time was $127 \pm 31 \mathrm{~min}$ vs. $107 \pm 46 \mathrm{~min}, p=1.00$. See Supplementary Table S3 for more details.

Untreated

Alteplase-treated

Apixaban-pretreated

Apixaban-pretreated + alteplase-treated

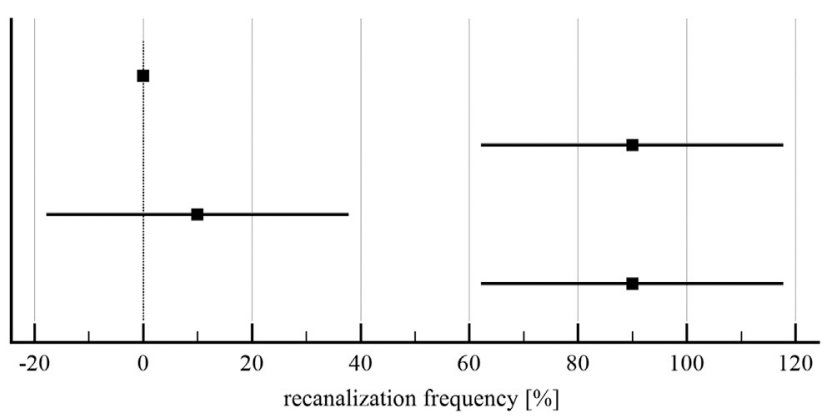

FIGURE 4 | Flow model: recanalization frequency of in vitro vessel. The forest plot shows mean values (square) and $95 \%$ confidence interval (whiskers). $n=5$. Results demonstrate that alteplase treatment provided efficient thrombolysis, e.g. documented as greater recanalization frequency compared to untreated group (90 \pm $22 \%$ vs. $0 \pm 0 \%, p<0.01$ ). For clots with and without apixaban, alteplase-induced lysis did not differ, e.g., recanalization frequency was $90 \pm 22 \%$ vs. $90 \pm 22 \%, p=1.00$. See Supplementary Table S3 for more details.

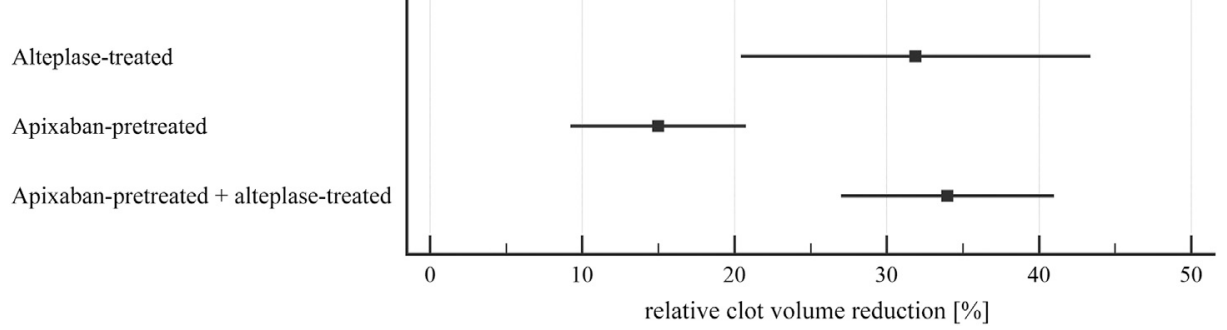

FIGURE 5 |Flow model: clot lysis expressed as relative clot volume reduction. The forest plot shows mean values (square) and 95\% confidence interval (whiskers). $n=9-10$. Results demonstrate that alteplase treatment provided efficient thrombolysis, e.g., documented as greater clot volume reduction compared to untreated group (32 $\pm 15 \%$ vs. $14 \pm 15 \%, p=0.02$ ). For clots with and without apixaban, alteplase-induced lysis did not differ, e.g., clot volume reduction was $34 \pm 10 \%$ vs. $32 \pm 15 \%$, $p=1.00$. See Supplementary Table S3 for more details.

in vitro studies with NOACs (apixaban and rivaroxaban) (Varin et al., 2013; Carter et al., 2018), we have not observed enhancement of thrombolysis by apixaban. Such observation is largely attributable to the therapeutic level of alteplase $\left(\sim 1 \mathrm{mg} \mathrm{L}^{-1}\right)$, which disfavor the molecular basis of the positive effect of a NOAC on plasminogen activation by alteplase (see detailed discussion below).

More importantly, in the flow model there was no influence of apixaban on alteplase efficiency as documented by four previously mentioned metrics: recanalization time, 


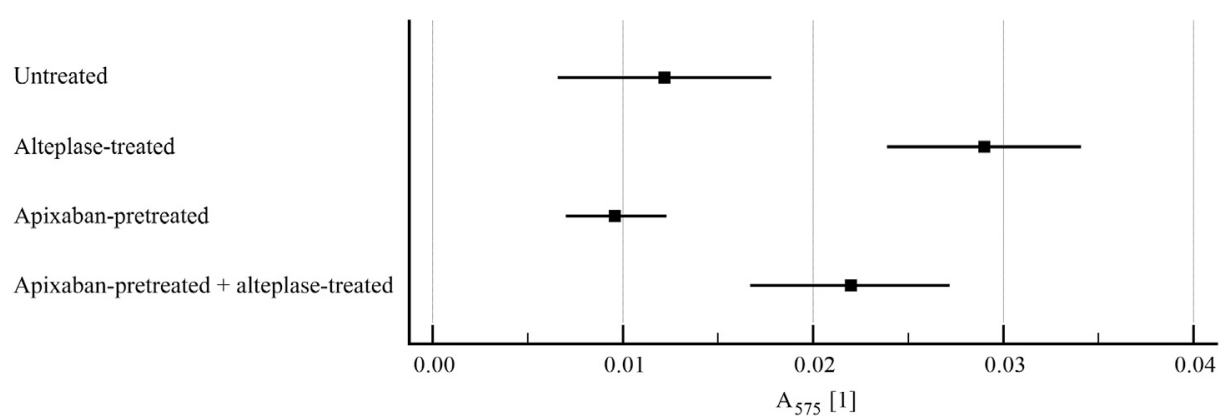

FIGURE 6 | Flow model: clot lysis expressed as red blood cell release into incubation media. The forest plot shows mean values (square) and 95\% confidence interval (whiskers). $n=9-10$. Results demonstrate that alteplase treatment provided efficient thrombolysis, e.g. documented as greater red blood cell release compared to untreated group $(0.029 \pm 0.007$ vs. $0.012 \pm 0.007, p<0.01)$. For clots with and without apixaban, alteplase-induced lysis marginally increased red blood cell release for clots without apixaban (0.022 \pm 0.007 vs. $0.029 \pm 0.007, p=0.16)$. See Supplementary Table S3 for more details.

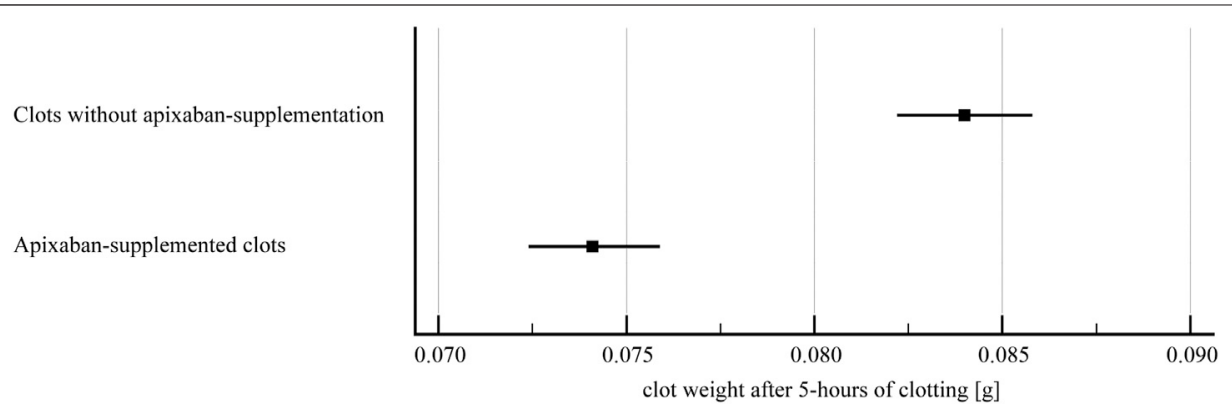

FIGURE 7 | Clot weight after 5-h of clotting. The forest plot shows mean values (square) and 95\% confidence interval (whiskers). $n=142$ (clots without apixaban), $n=137$ (apixaban-supplemented clots). Results demonstrate that apixaban supplementation provided lower clot weight after 5-h of clotting compared to clots without apixaban (0.07 $\pm 0.01 \mathrm{~g}$ vs. $0.08 \pm 0.01 \mathrm{~g}, p<0.01)$.

recanalization frequency and clot volume reduction and $\mathrm{RBC}$ release. Apixaban alone did not affect these characteristics as well. The obtained data are in agreement with the more simplistic static model. Compared to the static model, the flow model reflected clinical scenario better, because it included hemodynamic factors. Expectation was that the hydromechanical forces and resulting interstitial flow present in this system should allow better penetration of alteplase into the clot as previously reported (Diamond, 1999). To our knowledge there was no previous study documenting how apixaban can influence alteplase efficiency in an in vitro flow model, though the anticoagulant properties of apixaban were assayed under flow conditions in recent studies (Hosokawa et al., 2014; Sugihara et al., 2016; Pujadas-Mestres et al., 2017).

Previous experimental data, both in vitro and clinically indicated a positive effect of NOACs on thrombolysis, which is contrary to our finding. These studies however used alteplase at the endogenous level $\left(\sim 1 \mu \mathrm{g} \mathrm{L}{ }^{-1}\right)$ (Varin et al., 2013; Carter et al., 2018 ) or studied impact of NOACs on endogenous thrombolysis (Farag et al., 2016; Spinthakis et al., 2019). The therapeutic dosage, however, resulted in concentration which is about three orders of magnitude higher $\left(1.3 \mathrm{mg} \mathrm{L}^{-1}\right.$ in this study). Massive activation of plasminogen by such high concentration of alteplase can overwhelm NOAC-mediated enhancement of slow initial activation of plasminogen on intact fibrin fibers (Talbot et al., 2013; Carter et al., 2018). That is why NOACs can enhance thrombolysis at endogenous levels of alteplase, as observed in previous studies (Varin et al., 2013; Farag et al., 2016; Lau et al., 2016; Carter et al., 2018; Spinthakis et al., 2019); but is excluded at therapeutic level of alteplase as presented in this study. Accordingly, our finding that apixaban at clinically relevant concentration did not change the efficiency of alteplase in vitro corresponds with recent clinical meta-analyses. They indicated that apixaban pretreatment did not increase efficiency of alteplase in ischemic stroke patients. The combination of apixaban pretreatment with subsequent thrombolytic therapy with alteplase may be safe for selected patients; however, more data is needed to determine risk of bleeding (Tsivgoulis and Safouris, 2017; Shahjouei et al., 2020; Tsivgoulis et al., 2021).

A possible limitation of our study is that we used blood from healthy donors. In clinical practice patients suffer from comorbidities. With the exception of diabetes, these comorbidities do not directly affect function of alteplase (Zangerle et al., 2007). Hence, we do not expect major differences in results that would be obtained using blood from 
patients. Another limitation of the presented in vitro models is the inability to study intracranial bleeding, which would be a clinical concern if alteplase was administered to patients treated with apixaban.

In conclusion, our data from two different in vitro models, static and flow, consistently indicated no effect of apixaban on thrombolysis and recanalization induced by therapeutic levels of alteplase. We used different metrics and documented that alteplase had a suitably measurable effect, which supports the validity of our findings. Our data thus support current clinical practice that patients are not treated with alteplase if they were previously medicated with apixaban; due to no positive impact on thrombolysis and recanalization but uncertain risk of bleeding.

\section{DATA AVAILABILITY STATEMENT}

The original data presented in the study are included in Supplementary Material, further inquiries can be directed to the corresponding author.

\section{ETHICS STATEMENT}

The studies involving human participants were reviewed and approved by Ethical Committee, St. Anne's University Hospital Brno. The patients/participants provided their written informed consent to participate in this study.

\section{REFERENCES}

Acheampong, P., and Ford, G. A. (2012). Pharmacokinetics of alteplase in the treatment of ischaemic stroke. Expert Opin. Drug Metab. Toxicol. 8 (2), 271-281. doi:10.1517/17425255.2012.652615

Artang, R., Anderson, M., Riley, P., and Nielsen, J. D. (2017). Assessment of the effect of direct oral anticoagulants dabigatran, rivaroxaban, and apixaban in healthy male volunteers using a thrombin generation assay. Res. Pract. Thromb. Haemost. 1 (2), 194-201. doi:10.1002/rth2.12044

Byon, W., Garonzik, S., Boyd, R. A., and Frost, C. E. (2019). Apixaban: A Clinical Pharmacokinetic and Pharmacodynamic Review. Clin. Pharmacokinet. 58 (10), 1265-1279. doi:10.1007/s40262-019-00775-Z

Carter, R. L. R., Talbot, K., Hur, W. S., Meixner, S. C., Van Der Gugten, J. G., Holmes, D. T., et al. (2018). Rivaroxaban and apixaban induce clotting factor Xa fibrinolytic activity. J. Thromb. Haemost. 16 (11), 2276-2288. doi:10.1111/ jth.14281

Chao, Y. T., Hu, C. J., and Chan, L. (2019). Thrombolysis in an acute ischemic stroke patient with rivaroxaban anticoagulation: A case report. Medicine (Baltimore) 98 (8), e14560-3. doi:10.1097/MD.0000000000014560

Connolly, S. J., Eikelboom, J., Joyner, C., Diener, H. C., Hart, R., Golitsyn, S., et al. (2011). Apixaban in Patients with Atrial Fibrillation. N. Engl. J. Med. 364, 806-817. doi:10.1056/NEJMoa1007432

Connolly, S. J., Ezekowitz, M. D., Yusuf, S., Eikelboom, J., Oldgren, J., Parekh, A., et al. (2009). Dabigatran versus warfarin in patients with atrial fibrillation. $N$. Engl. J. Med. 361, 1139-1151. doi:10.1056/NEJMoa0905561

Diamond, S. L. (1999). Engineering design of optimal strategies for blood clot dissolution. Annu. Rev. Biomed. Eng. 1, 427-462. doi:10.1146/annurev.bioeng.1.1.427

Elnager, A., Abdullah, W. Z., Hassan, R., Idris, Z., Wan Arfah, N., Sulaiman, S. A., et al. (2014). In Vitro Whole Blood Clot Lysis for Fibrinolytic Activity Study

\section{AUTHOR CONTRIBUTIONS}

All authors contributed significantly to the manuscript. ST: Experimental work-static model, experimental work management, data processing, data analysis, data revisions, data presentation, text writing, MP: Experimental work-static model, data processing, PK: Experimental work-flow model, data processing, SG: Text writing, JV: Experimental work supervision, research idea, data interpretation, text writing, LK: Critical evaluation, funding, RM: Research idea, critical evaluation, funding, data interpretation.

\section{FUNDING}

This work was supported by the Pfizer Inc. through a competitive grant from the BMS/Pfizer European Thrombosis Investigator Initiated Research Program (ERISTA). ST was supported by Brno Ph.D. Talent Scholarship (Brno City Municipality). MP was supported by project MUNI/C/0030/2020 (Masaryk University). JV, LK and RM were supported by the European Regional Development Fund-Project INBIO (No. CZ.02.1.01/ 0.0/0.0/16_026/0008451).

\section{SUPPLEMENTARY MATERIAL}

The Supplementary Material for this article can be found online at: https://www.frontiersin.org/articles/10.3389/fphar.2021.740930/ full\#supplementary-material

Using D-Dimer and Confocal Microscopy. Adv. Hematol. 2014, 814684-814688. doi:10.1155/2014/814684

Escolar, G., Fernandez-Gallego, V., Arellano-Rodrigo, E., Roquer, J., Reverter, J. C., Sanz, V. V., et al. (2013). Reversal of apixaban induced alterations in hemostasis by different coagulation factor concentrates: significance of studies in vitro with circulating human blood. PLoS One 8 (11), e78696. doi:10.1371/ journal.pone.0078696

Farag, M., Niespialowska-Steuden, M., Okafor, O., Artman, B., Srinivasan, M., Khan, A., et al. (2016). Relative effects of different non-vitamin K antagonist oral anticoagulants on global thrombotic status in atrial fibrillation. Platelets 27 (7), 687-693. doi:10.3109/09537104.2016.1158402

Granger, C. B., Alexander, J. H., McMurray, J. J., Lopes, R. D., Hylek, E. M., Hanna, M., et al. (2011). Apixaban versus Warfarin in Patients with Atrial Fibrillation. N. Engl. J. Med. 365, 981-992. doi:10.1056/NEJMoa1107039

Harpaz, D., Chen, X., Francis, C. W., Marder, V. J., and Meltzer, R. S. (1993). Ultrasound enhancement of thrombolysis and reperfusion in vitro. J. Am. Coll. Cardiol. 21 (6), 1507-1511. doi:10.1016/0735-1097(93)90331-t

Hosokawa, K., Ohnishi, T., Sameshima, H., Miura, N., Koide, T., Maruyama, I., et al. (2014). Comparative Evaluation of Direct Thrombin and Factor Xa Inhibitors with Antiplatelet Agents under Flow and Static Conditions: An in Vitro Flow Chamber Model. PLoS One 9 (1), e86491. doi:10.1371/ journal.pone.0086491

Ishihara, H., Torii, H., Imoto, H., Oka, F., Sadahiro, H., and Suzuki, M. (2014). Intravenous thrombolysis with recombinant tissue plasminogen activator in a stroke patient treated with rivaroxaban. J. Stroke Cerebrovasc. Dis. 23 (10), e457-459. doi:10.1016/j.jstrokecerebrovasdis.2014.07.008

Jiang, X., Crain, E. J., Luettgen, J. M., Schumacher, W. A., and Wong, P. C. (2009). Apixaban, an oral direct factor Xa inhibitor, inhibits human clot-bound factor Xa activity in vitro. Thromb. Haemost. 101 (4), 780-782. doi:10.1160/th08-070486 
Jin, C., Huang, R. J., Peterson, E. D., Laskowitz, D. T., Hernandez, A. F., Federspiel, J. J., et al. (2018). Intravenous tPA (Tissue-type Plasminogen Activator) in Patients with Acute Ischemic Stroke Taking Non-vitamin K Antagonist Oral Anticoagulants Preceding Stroke. Stroke 49 (9), 2237-2240. doi:10.1161/ STROKEAHA.118.022128

Kim, Y. D., Nam, H. S., Kim, S. H., Kim, E. Y., Song, D., Kwon, I., et al. (2015). Time-Dependent Thrombus Resolution after Tissue-type Plasminogen Activator in Patients with Stroke and Mice. Stroke 46 (7), 1877-1882. doi:10.1161/STROKEAHA.114.008247

Lau, Y. C., Xiong, Q., Shantsila, E., Lip, G. Y., and Blann, A. D. (2016). Effects of Nonvitamin K Antagonist Oral Anticoagulants on Fibrin Clot and Whole Blood Clot Formation, Integrity and Thrombolysis in Patients with Atrial Fibrillation. J. Thromb. Thrombolysis 42 (4), 535-544. doi:10.1007/s11239-016-1399-3

Patel, M. R., Mahaffey, K. W., Garg, J., Pan, G., Singer, D. E., Hacke, W., et al. (2011). Rivaroxaban versus Warfarin in Nonvalvular Atrial Fibrillation. N. Engl. J. Med. 365, 883-891. doi:10.1056/NEJMoa1009638

Pinto, D. J., Orwat, M. J., Koch, S., Rossi, K. A., Alexander, R. S., Smallwood, A., et al. (2007). Discovery of 1-(4-methoxyphenyl)-7-oxo-6-(4-(2-oxopiperidin-1Yl)phenyl)-4,5,6,7-tetrahydro-1h-pyrazolo[3,4-c]pyridine-3-carboxamide (apixaban, BMS-562247), a highly potent, selective, efficacious, and orally bioavailable inhibitor of blood coagulation factor Xa. J. Med. Chem. 50 (22), 5339-5356. doi:10.1021/jm070245n

Prasad, S., Kashyap, R. S., Deopujari, J. Y., Purohit, H. J., Taori, G. M., and Daginawala, H. F. (2006). Development of an in vitro model to study clot Lysis activity of thrombolytic drugs. Thromb. J. 4 (14), 14-4. doi:10.1186/1477-95604-14

Pujadas-Mestres, L., Lopez-Vilchez, I., Arellano-Rodrigo, E., Reverter, J. C., LopezFarre, A., Diaz-Ricart, M., et al. (2017). Differential Inhibitory Action of Apixaban on Platelet and Fibrin Components of Forming Thrombi: Studies with Circulating Blood and in a Platelet-Based Model of Thrombin Generation. PLoS One 12 (2), e0171486. doi:10.1371/journal.pone.0171486

Šaňák, D. (2018). Intravenous Thrombolysis in Patients with Acute Ischemic Stroke after a Reversal of Dabigatran Anticoagulation with Idarucizumab: A Real-World Clinical Experience. J. Stroke Cerebrovasc. Dis. 27 (9), 2479-2483.

Seiffge, D. J., Hooff, R. J., Nolte, C. H., Béjot, Y., Turc, G., Ikenberg, B., et al. (2015). Recanalization therapies in acute ischemic stroke patients: impact of prior treatment with novel oral anticoagulants on bleeding complications and outcome. Circulation 132 (13), 1261-1269. doi:10.1161/CIRCULATIONAHA.115.015484

Shahjouei, S., Tsivgoulis, G., Goyal, N., Sadighi, A., Mowla, A., Wang, M., et al. (2020). Safety of Intravenous Thrombolysis Among Patients Taking Direct Oral Anticoagulants: A Systematic Review and Meta-Analysis. Stroke 51 (2), 533-541. doi:10.1161/STROKEAHA.119.026426

Spinthakis, N., Gue, Y., Farag, M., Srinivasan, M., Wellsted, D., Arachchillage, D. R. J., et al. (2019). Apixaban enhances endogenous fibrinolysis in patients with atrial fibrillation. Europace 21 (9), 1297-1306. doi:10.1093/europace/euz176

Sugihara, H., Idemoto, Y., Kuwano, T., Nagata, Y., Morii, J., Sugihara, M., et al. (2016). Evaluation of the Antithrombotic Effects of Rivaroxaban and Apixaban
Using the Total Thrombus-Formation Analysis System ${ }^{\circledR}$ : In Vitro and Ex Vivo Studies. J. Clin. Med. Res. 8 (12), 899-907. doi:10.14740/jocmr2773w

Sutton, J. T., Ivancevich, N. M., Perrin, S. R., Vela, D. C., and Holland, C. K. (2013). Clot Retraction Affects the Extent of Ultrasound-Enhanced Thrombolysis in an Ex Vivo Porcine Thrombosis Model. Ultrasound Med. Biol. 39 (5), 813-824. doi:10.1016/j.ultrasmedbio.2012.12.008

Talbot, K., Meixner, S. C., and Pryzdial, E. L. (2013). Proteolytic Modulation of Factor Xa-antithrombin Complex Enhances Fibrinolysis in Plasma. Biochim. Biophys. Acta 1834 (6), 989-995. doi:10.1016/j.bbapap.2013.02.007

Tsivgoulis, G., Kargiotis, O., De Marchis, G., Kohrmann, M., Sandset, E. C., Karapanayiotides, T., et al. (2021). Off-label use of intravenous thrombolysis for acute ischemic stroke: a critical appraisal of randomized and real-world evidence. Ther. Adv. Neurol. Disord. 14, 1756286421997368. doi:10.1177/ 1756286421997368

Tsivgoulis, G., and Safouris, A. (2017). Intravenous Thrombolysis in Acute Ischemic Stroke Patients Pretreated with Non-vitamin K Antagonist Oral Anticoagulants: An Editorial Review. Stroke 48 (7), 2031-2033. doi:10.1161/ STROKEAHA.117.017206

Varin, R., Mirshahi, S., Mirshahi, P., Klein, C., Jamshedov, J., Chidiac, J., et al. (2013). Whole Blood Clots Are More Resistant to Lysis Than Plasma Clots-Greater Efficacy of Rivaroxaban. Thromb. Res. 131 (3), e100-9. doi:10.1016/ j.thromres.2012.11.029

Zangerle, A., Kiechl, S., Spiegel, M., Furtner, M., Knoflach, M., Werner, P., et al. (2007). Recanalization after Thrombolysis in Stroke Patients: Predictors and Prognostic Implications. Neurology 68 (1), 39-44. doi:10.1212/ 01.wnl.0000250341.38014.d2

Conflict of Interest: This study received funding from the Pfizer Inc. through a competitive grant from the BMS/Pfizer European Thrombosis Investigator Initiated Research Program (ERISTA). The funder was not involved in the study design, collection, analysis, interpretation of data and the writing of this article. The submission for publication was approved by the funder. All authors declare no other competing interests.

Publisher's Note: All claims expressed in this article are solely those of the authors and do not necessarily represent those of their affiliated organizations, or those of the publisher, the editors and the reviewers. Any product that may be evaluated in this article, or claim that may be made by its manufacturer, is not guaranteed or endorsed by the publisher.

Copyright (C) 2021 Thalerová, Pešková, Kittová, Gulati, Víteček, Kubala and Mikulik. This is an open-access article distributed under the terms of the Creative Commons Attribution License (CC BY). The use, distribution or reproduction in other forums is permitted, provided the original author(s) and the copyright owner(s) are credited and that the original publication in this journal is cited, in accordance with accepted academic practice. No use, distribution or reproduction is permitted which does not comply with these terms. 